\title{
Influence of outpatient total knee arthroplasty compared to inpatient surgery on medical and economic outcomes
}

\author{
Vincent Tomasi, Alex Demurie, Ignace Ghijselings, Olivier Cornu, Hans Van Den Wyngaert
}

From the Department of Orthopaedic Surgery, AZ Alma, Eeklo, Belgium

Firstly, this study compared the rate of readmission after a total knee arthroplasty between selected outpatients (no hospitalization, directly sent home after surgery) and inpatients ( 3 days hospitalization) at 6 weeks. Secondly, it examined the mobility and the complications in the two groups after the same period of time.

The rate of readmission, complications and knee mobility of 32 outpatients ( $M$-age : 61 years $\pm 10 ; 10$ females), were compared against those of 32 birthmatched inpatients $(M$-age : 64 years $\pm 8.6 ; 10$ females).

No patient was re-admitted in either group. Postsurgical complications included one hematoma resorbed at 6 weeks in the outpatient group and three joint effusions in the inpatient group. There were no instances of deep venous thrombosis, failure of primary fixation, infection, or wound dehiscence. Knee mobility was identical between the two groups.

This is the first study to compare inpatient and outpatient total knee arthroplasty in a Belgian setting. Our study suggests that day-care total knee arthroplasty in selected patients is possible without increasing the rate of re-admission and complications, and without affecting the mobility at 6 weeks. However, the Belgian financial incentives do not seem to currently promote this surgical approach. These results should be confirmed with a larger sample to define the adequate length of stay after a total knee arthroplasty.

Keywords : Total knee arthroplasty ; outpatient ; fasttrack program ; complications ; discharge criteria.

No benefits or funds were received in support of this study. None of the authors have a conflict of interest.

\section{INTRODUCTION}

Fast track (FT) in total knee arthroplasty (TKA) is defined as a hospitalization which provides the best possible evidence-based treatment and a maximum hospital stay of 3 days (1). Today, FT procedure is recommended for most patients who need a TKA (1), considering that such an optimisation program can only be achieved with the support of a special multidisciplinary team (2).

FT in TKA aims to reduce morbidity, mortality, and functional convalescence while allowing a reduced length of stay (LOS) and high patient satisfaction (3). Due to the optimisation of preoperative, perioperative and post-operative care, outpatient TKA can be seen as the ultimate goal of FT surgery. However, it requires focus on both the patient's comorbidities and home environment

\footnotetext{
Vincent Tomasi ${ }^{1} \mathrm{MD}$,

Alex Demurie ${ }^{2} \mathrm{MD}$,

Ignace Ghijselings ${ }^{2} \mathrm{MD}$,

- Olivier Cornu ${ }^{1} \mathrm{MD}$ PhD,

- Hans Van Den Wyngaert ${ }^{2}$ MD

'Division of Orthopaedic Surgery, Department of Surgery, Cliniques Universitaires Saint-Luc, Brussels, Belgium.

${ }^{2}$ Department of Orthopaedic Surgery, AZ Alma, Eeklo, Belgium.

Correspondence : Vincent Tomasi, 24 rue Niemegeers, 1950 Kraainem. Phone : +32 4773220 36, Fax : +32 276489 04, ORCID identifier: 0000-0002-0434-7381.

Email : Vincent.Tomasi@outlook.com

- 2021, Acta Orthopædica Belgica.
} 
to ensure that surgeon and patient expectations are aligned (4). Recent proposals for outpatient surgery have raised the question of the risks and benefits of shorter LOS (5-7).

The rehospitalization and complication rates in outpatients discharged directly to their homes after TKA, have been scarcely described. This retrospective comparative study aims to determine whether FT outpatients discharged directly to their homes on the day of surgery have comparable follow-up outcomes at 6 weeks after discharge as inpatients who stay in hospital for 3 days. Selection criteria for FT TKA and economic features relative to same-day surgery are discussed.

\section{MATERIALS AND METHODS}

The data of patients who underwent TKA were identified from the database of AZ Alma hospital (Eeklo, Belgium). All patients were referred to 3 surgeons (Dr. Alex Demurie, Dr. Ignace Ghijselings, Dr. Hans Van den Wyngaert) who used the same patella in place balancing (PIPB) surgical technique with a 'no tissue release' philosophy (8) (Lazi-rush ${ }^{\circledR}$ technique).

A retrospective review was performed. Patient information such as date of birth, gender, medical and functional status prior and after surgery, and LOS were available in the database. The study included adult patients who underwent primary and elective TKA, with a LOS of 3 days (inpatients) or 0 day (less than 10 hours; outpatients). Further, the included patients had no comorbidities (e.g., diabetes, heart disease, renal or lung failure, use of anticoagulation, infection, systemic disease (corresponding to an American Society of Anaesthesiologists (ASA) score $<$ III)). With respect to the outpatients, additional inclusion criteria were a BMI of $<35 \mathrm{~kg} /$ $\mathrm{m}^{2}$ and the return home directly following surgery. Between 2008 and 2018, 32 outpatients with TKA were identified and paired with 32 birth- and gendermatched inpatients with TKA (Table I).

The principal variable was the rehospitalization rate, while the other variables included mobility (based on categories A-B-C), pre and postoperative hemoglobin levels and the complication rate (failure of primary fixation, presence of deep venous throm-
Table I. - Demographics

\begin{tabular}{|l|c|c|}
\hline & $\begin{array}{c}\text { Group } \\
\text { outpatients }\end{array}$ & $\begin{array}{c}\text { Group } \\
\text { inpatients }\end{array}$ \\
\hline Number of TKAs & 32 & 32 \\
\hline $\begin{array}{l}\text { Age at the time of } \\
\text { surgery: min-max (mean } \\
\text { and standard error) }\end{array}$ & $43-77(61 \pm 10)$ & $48-85(64 \pm 8.6)$ \\
\hline Male/Female & $22 \mathrm{M} / 10 \mathrm{~F}$ & $22 \mathrm{M} / 10 \mathrm{~F}$ \\
\hline
\end{tabular}

Note : some patients in the study underwent two successive TKA (9 outpatients and 2 inpatients)

bosis (DVT), clinical signs of infection or wound dehiscence, pain and knee joint effusion) in the two groups at 6 weeks post-surgery.

The knee mobility after operation was categorized into three groups : $\mathrm{A}=$ flexion above $110^{\circ}$ without extension deficit; $\mathrm{B}=$ flexion between $100^{\circ}-110^{\circ}$ without extension deficit $; \mathrm{C}=$ flexion under $100^{\circ}$ and/or extension deficit.

Preoperative blood samples were taken within 3 months before surgery, and post-operative samples were collected within 6 weeks (from day 0 to day 37) after surgery for outpatients and within 2 days for inpatients.

The preoperative protocol included general anaesthesia (for maximal muscle relaxation, blood pressure control, and rapid mobilisation after a few hours post-surgery, without the need of a bladder catheter), pre-medication (cefazoline $2 \mathrm{~g}$, tranexamic acid $2 \times 500 \mathrm{mg}$, parecoxib $40 \mathrm{mg}$, tramadol 1-2 ampoule, methylprednisolone 40mg, magnesium sulfate, ondansetron), and antalgics during the anesthesia (paracetamol $2 \times 1 \mathrm{~g}$ and $20 \mathrm{mg}$ piritramide). All TKAs were performed with a far medial subvastus approach, local infiltration anesthesia of ripovacaïne and adrenaline, and without the use of a drain or tourniquet. A Triathlon ${ }^{\circledR}$ Total Knee System (Stryker ${ }^{\circledR}$, Mahwah, NJ, USA) was used with cement (Palacos ${ }^{\circledR}$ or Optipac $\AA$ ) for both femoral and tibial components, and a cruciate retaining $(\mathrm{CR})$ or condylar stabilizing $(\mathrm{CS})$ tibial X3 highly crosslinked polyethylene bearing insert was inserted. After surgery, patients were transferred to the post anaesthesia care unit (PACU) where ice was placed alongside the wound, no pain pump was used but piritramide IV was administered. The patients could ambulate and mobilize the knee in 
Table II. - Recommendations of the American Association of Hip and Knee Surgeons regarding the criteria for a safe outpatient stay in the context of a TKA surgery, 10 April 2018

Criteria of outpatient discharge after a TKA

An outpatient stay is deemed safe when the patient may be discharged to home on the day of surgery based on the presence of the following factors:

1. The patient is able to ambulate independently

2. The patient has adequate pain control on oral pain medications

3. The patient tolerates oral intake

4. The patient has the ability to void freely

5. The patient is hemodynamically stable

6. In the opinion of the physician the patient does not require laboratory monitoring

7. The patient does not have a medical condition that requires management in a hospital setting

$90^{\circ}$ flexion the evening of the surgery. Painkillers were used (paracetamol 1 $\mathrm{g}$ IV or per os, etoricoxib $120 \mathrm{mg} /$ day if not contraindicated, tramadol per os if required) and enoxaparine 4000UI was introduced 24 hours after surgery.

The American Association of Hip and Knee Surgeons (AAHKS) criteria for discharge (9) were applied to all patients; the patients stayed in the hospital until those criteria were fulfilled (Table II). The outpatients were discharged directly to their homes (rather than to a nursing facility or a revalidation center) and had access to a mechanic trainer to exercise the operated knee (two sessions a day). The physiotherapist and the nurse visited the patients three times a week. The inpatients had the same discharge criteria, however they received physiotherapy and nurse care each day during their 3-day hospitalization.

At 6 weeks post-surgery, a follow-up visit was organized consisting of a clinical exam and knee radiography.

A between-group comparison of the categorical variable knee mobility relied on a non-inferiority testing (IBM SPSS statistics). The confidence interval was compared to the non-inferiority limit, which was set to $-20 \%$ in order to define whether the outpatient group could be considered as noninferior to the inpatient group on the knee mobility variable.

The research protocol followed the tenets of the Declaration of Helsinki and was approved by the AZ Alma Ethics Committee under registration number EC/EH/20190927. There was no funding and no conflict of interest in this study.

\section{RESULTS}

Thirty-two outpatients with TKA and thirty-two inpatients with TKA were selected according to the inclusion criteria described above. Demographic data are displayed in Table I and Fig. 1.

At 6 weeks post-surgery, no rehospitalization neither in the inpatient group nor in the outpatient group was observed.

Regarding complications at 6 weeks post-surgery, no failure of primary fixation upon Rx control, no signs of wound dehiscence (open wound, presence of pus), no clinical signs of infection (erythema, warmth, pain and joint effusion), and no signs of DVT after echography or medical exam were reported in both groups. Pain was present in all patients in both groups but was controlled with painkillers (ice, paracetamol, non-steroidal anti-inflammatory drugs, tramadol). Three inpatients pre-

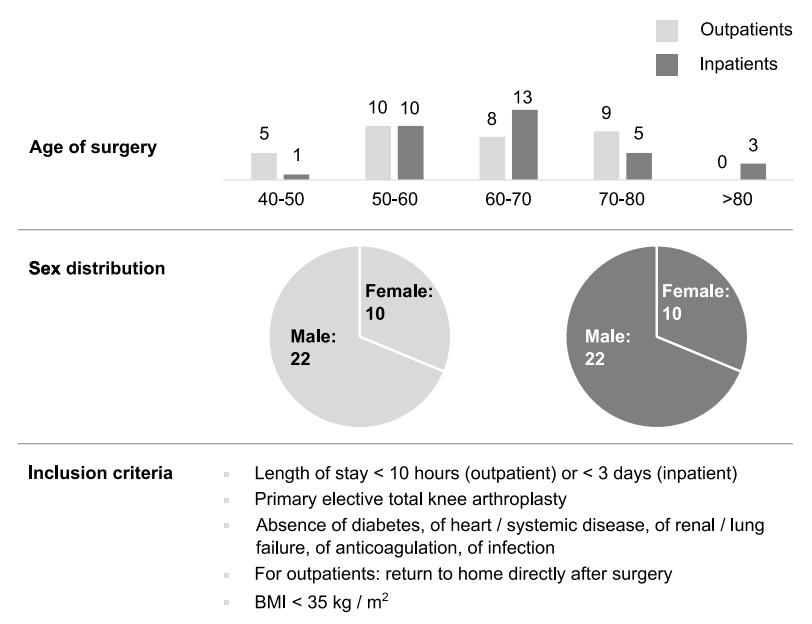

Fig. 1. - Demographics and criteria of inclusion of outpatients and inpatients. 


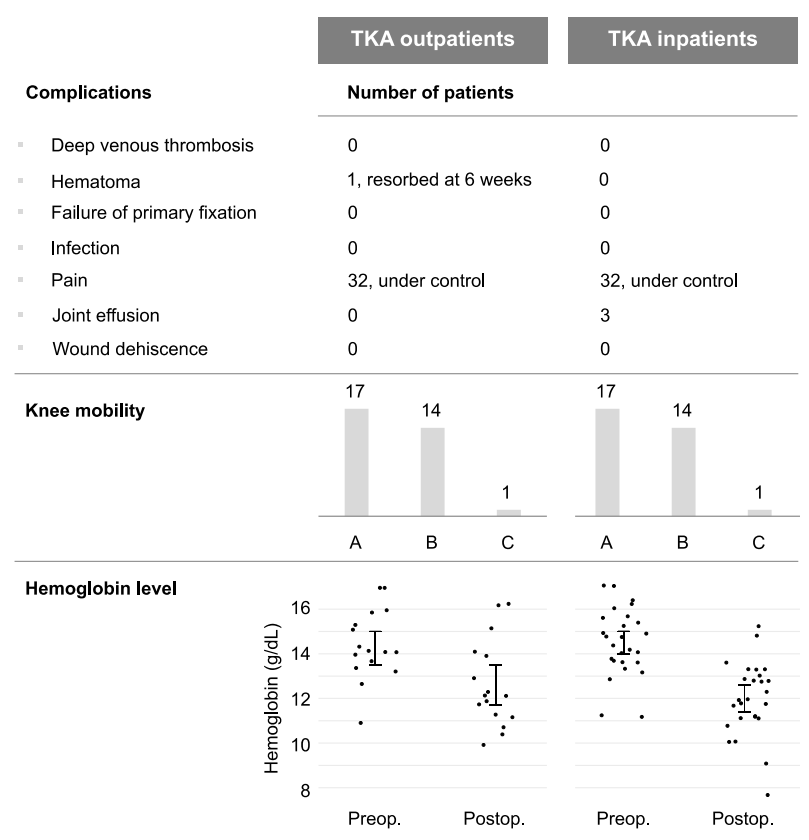

Fig. 2. - In outpatients and inpatients, post-surgical complications, knee mobility $\left(\mathrm{A}=\right.$ flexion above $110^{\circ}$ without extension deficit; $\mathrm{B}=$ flexion between $100^{\circ}-110^{\circ}$ without extension deficit; $\mathrm{C}=$ flexion under $100^{\circ}$ and/or extension deficit) and preoperative / postoperative hemoglobin, with error bars representing $95 \%$ confidence interval.

sented with a significant joint effusion. In the outpatient group, one patient had a post-operative periprosthetic bleeding with hematoma which was conservatively treated and resorbed at 6 weeks (Fig. 2).

Mean preoperative hemoglobin levels for outpatients $(\mathrm{n}=16)$ was $14,3 \mathrm{~g} / \mathrm{dL}(95 \% \mathrm{CI}:[13,5-15])$ and $14,5 \mathrm{~g} / \mathrm{dL}(95 \% \mathrm{CI}:[13,9-15])$ for inpatients $(\mathrm{n}=27)$. Mean post-operative hemoglobin levels for outpatients $(\mathrm{n}=16)$ was $12,6 \mathrm{~g} / \mathrm{dL}(95 \% \mathrm{CI}$ : [11,7-13,5]) and $11,9 \mathrm{~g} / \mathrm{dL}(95 \% \mathrm{CI}:[11,3-12,6])$ for inpatients $(\mathrm{n}=26)$ (Fig. 2).

In terms of knee mobility, 17 patients were categorized as A in both groups, while 14 were categorized as B in both groups. In the outpatient group, one patient had a flexion under $100^{\circ}$ at 6 weeks (category $\mathrm{C}$ ). In the inpatient group, one patient had an extension deficit at 6 weeks (category C) (Fig. 2).

The confidence interval (CI) for the betweengroup comparison of the categorical variable knee mobility assessed as category A was $[-0,693$ to $0,693]$. As the lower limit of the confidence interval
$(-0,693)$ was below the non-inferiority limit set by protocol at $-20 \%$, knee mobility in the outpatient group cannot be considered as non-inferior to knee mobility in the inpatient group at 6 weeks postsurgery. Statistics on categories B and C were deemed non-relevant.

\section{DISCUSSION}

The present study showed that outpatients can have comparably satisfactory follow-up results at 6 weeks of discharge as inpatients who stay in hospital for 3 days. At 6 weeks, no rehospitalization was reported neither in the inpatient group nor in the outpatient group. At 6 weeks, no patient presented with complications with the exception of 1 hematoma in the outpatient group after one week (resorbed at 6 weeks) and 3 knee joint effusions in the inpatient group at 6 weeks. While present, pain was controlled with painkillers for every patient. The changes in hemoglobin levels suggested a slightly higher mean drop in the inpatient group $(-2,6 \mathrm{~g} / \mathrm{dL})$ versus the outpatient group $(-1,7 \mathrm{~g} / \mathrm{dL})$ what could reflect the time spreading of post-operative blood sampling in the outpatient group. The distribution of knee mobility results per category was similar in both groups, with a majority of patients classified as category A, and $97 \%$ of patients classified as categories A or B (31/32 in each group), confirming a good outcome in outpatients as in inpatients. Therefore, the knee mobility in the outpatient group statistically cannot be considered as non-inferior to the knee mobility in the inpatients group.

Our medical outcomes for FT TKA are generally aligned with results from several recently published favourable studies. However, literature on outpatient TKA has also provided mixed results.

In the Netherlands (10), a study showed that physical activity in daily life at 6 weeks after TKA was similar between outpatients and inpatients. In a French study (7), no significant difference was observed between two similar groups regarding the readmission rate on day $30(3,3 \%$ in the outpatient group and $4,9 \%$ in the inpatient group), the complication rate $(8,2 \%$ in the outpatient group and 7,5 in the inpatient group), and no revision was required in both groups. 
Other recent US studies reported favourable results in terms of immediate and short-term complications and readmissions (6), or return events at 90 days relating to health condition and not to the LOS (11). Other studies flagged some potential negative differences. Berger and colleagues showed in a first study (12) that properly selected outpatient TKA is feasible with no readmission, reoperation, or significant complication related to early discharge and might have decreased complications and increased patient satisfaction. However, another study by the same authors (13) highlighted that in an unselected group, outpatient TKA can be performed in a high percentage of patients, but readmission rate may be slightly higher. In another US trial (14), the readmission rate at 30 days was not statistically different, but the authors reported a higher rate of post-discharge blood transfusions in outpatients compared to inpatients, which could be a consequence of hidden continuous blood loss due to higher post-operative activity. Lovald and colleagues reported a slight increase in revision/ readmission and mortality risk for outpatients or patients with a LOS $<2$ days compared to patients with a longer LOS (15).

Using a different approach (aggregate analysis of data from a large private health insurance database from multiple US institutions), Arshi and colleagues showed that outpatients with TKA are associated with a higher risk of complications such as infection (at 6 months), knee stiffness (at 1 year), DVT (at 1 year) and component failure (at 1 year) (16). The authors noted a marked difference between single institution and multi-institution data that may be due to the fact that the high-volume centers were better equipped than the average U.S. institution to facilitate the comprehensive clinical pathways necessary for safe and effective discharge.

When comparing data from the recent literature, it appears that the success of the FT TKA is mainly defined by the perioperative patient management and the application of selection criteria. In our study, selection criteria included the following: patients needed to (i) be the first or second patient operated on the day, (ii) have no significant preoperative comorbidity, and (iii) have a social network (i.e., the patient could not live alone and could easily reach staff members).

Selection of patients for FT TKA is crucial for most authors $(2,4,19-22,5-7,10,14,16-18)$. Standard criteria are based on comorbidities, BMI, age, social network and ability to reach staff members. A low comorbidity level (similar to an ASA score < III) is a common recommendation, while other criteria are still debatable. Older age is expected to be associated with longer LOS because of increased comorbidities linked with age $(7,19,22)$. However, some authors showed no clear age limitation for FT TKA (4) and others did not use age criteria for outpatient selection (7). BMI limit as a selection criterion is debatable. Some authors pointed out that a BMI $>30$ is predictive of a negative outcome after TKA regarding infection and revision rate $(19,22)$. Others excluded patients with a BMI $>40$ (12). Some authors did not use BMI as exclusion criteria $(4,7)$. While Husted and colleagues found that female gender may increase LOS (23), a study by Crawford showed no LOS differences between males and females (17). Another important selection criteria relate to the patient social network and home environment, as well as the ability to easily reach staff members for advice $(4,12,22)$. Finally, there is a common agreement between authors for the need of multidisciplinary patient management to ensure a successful FT TKA $(2,4,6,10,14,19$ 21,24). This is based on patient and carer education, operation planning (early in the morning), optimal pain management, combined with ergotherapist, kinesitherapist, nutritionist and nursing support.

Recent projections suggest that the number of total joint arthroplasty procedures will greatly increase over the coming decades. With the potential to reduce costs, FT TKA is becoming an increasingly popular option $(4,10,12,17,18,21)$. A recent European publication supported the notion that a same day admission and discharge is possible for selected TKA patients, reporting a similar rate of readmission or adverse events, as well as proven benefit for the patient, surgeon and the health insurance system (20). Reducing LOS will decrease individual and societal costs only if there is no increased complications nor readmission rate. The effort of reducing LOS should 
not be at the expense of readmission, or visiting the emergency department which would increase the cost of care (1). In hospitals, protocols for outpatient surgery are cost effective only when the available beds are occupied by new patients $(4,10)$. A short LOS would lead to a substantial reduction in cost but the expenses related to follow-up at the patient's home, nursing facility or the rehabilitation centre are still an issue (5). Overall, two complementary strategies need to be considered to reduce patient cost of TKA, to shorten LOS and to minimize perioperative and post-operative complications.

Currently in Belgium, TKA is not recognized on the health authority list of funded outpatientsurgeries. In the case of an outpatient TKA for a patient below 75-years of age and with low comorbidity risks, the hospital would only receive a fixed fee for the fitting of the prosthesis, but no additional funding for bed occupancy in day hospital. On the contrary, in the case of an inpatient TKA for the same patient, the hospital would receive bed occupancy funding for 5 days. In practice, it is financially more advantageous for the hospital to keep a patient for 2 days in hospital after a TKA, even if that patient could have left the hospital safely on the day of the surgery. In conclusion, the current type of funding in Belgium is not favourable for the establishment of TKA in day hospitals and for FT protocols.

Our study has several limitations. Future studies on this topic should increase the number of patients, follow a prospective design, and collect more detailed data on BMI, pre- and post-hemoglobin values, and additional visits to the emergency room or other medical facilities.

This is the first study to compare inpatient and outpatient TKA results in a Belgian setting. It supports that a same day admission and discharge is possible for selected TKA patients when strict protocols are put in place. This should be to the benefit of the patient, the hospital and the health insurance system. However, it is recognized that in Belgium, the healthcare system does not incite outpatient TKA. More data are needed to promote change in the outpatient TKA funding by the Belgian health authorities.

\section{REFERENCES}

1. Rodriguez-Merchan EC. Pros and Cons of Fast-track Total Knee Arthroplasty. Int. J. Orthop. 2015 ; 2(3) : 270-9.

2. Kosev P, Sokolov T, Pavlova I, Valentinov B, Andonov J, Petrova N. Fast Track surgery in total knee arthroplasty - A review. J. IMAB. 2015 ; 21(3) : 837-9.

3. Husted H. Fast-track hip and knee arthroplasty : Clinical and organizational aspects. Acta Orthop. $2012 ; 83$ : 1-39.

4. Argenson JNA, Husted H, Lombardi A, Booth RE, Thienpont E. Global forum : An international perspective on outpatient surgical procedures for adulthip and knee reconstruction. J. Bone Jt. Surg. Am. 2016 ; 98(13) : e55.

5. Thienpont E, Lavand'Homme $\mathbf{P}$, Kehlet $\mathbf{H}$. The constraints on day-case total knee arthroplasty : The fastest fast track. Bone Jt J. 2015 ; 97-B(10 Suppl A) : 40-4.

6. Hoeffel DP, Daly PJ, Kelly BJ, Giveans MR. Outcomes of the First 1,000 Total Hip and Total Knee Arthroplasties at a Same-day Surgery Center Using a Rapid-recovery Protocol. JAAOS Glob. Res. Rev. 2019; 3(3) : e022.

7. Cassard X, Garnault V, Corin B, Claverie D, Murgier J. Outpatient total knee arthroplasty: Readmission and complication rates on day 30 in 61 patients. Orthop. Traumatol. Surg. Res. 2018 ; 104 : 967-70.

8. Ettinger M, Calliess T, Demurie A, Van den Wyngaert H, Ghijselings I. Patella in Place Balancer : Technique for total knee arthroplasty. Orthopade. $2015 ; 44(4)$ : 269-74.

9. American Association of Hip and Knee Surgeons (AAHKS). Outpatient TKA Patient Selection Criteria. www.aaos. org/globalassets/advocacy/issues/aahks-outpatient-tkaselection-criteria-4-18-2018.pdf. 2018.

10. Schotanus MGM, Bemelmans YFL, Grimm B, Heyligers IC, Kort NP. Physical activity after outpatient surgery and enhanced recovery for total knee arthroplasty. Knee Surgery, Sport Traumatol. Arthrosc. 2017 ; 25(11) : 3366-71.

11. Rossman SR, Reb CW, Danowski RM, Maltenfort MG, Mariani JK, Lonner JH. Selective Early Hospital Discharge Does Not Increase Readmission but Unnecessary Return to the Emergency Department Is Excessive Across Groups After Primary Total Knee Arthroplasty. $J$. Arthroplasty. 2016 ; 31(6) : 1175-8.

12. Berger RA, Sanders S, Gerlinger T, Della Valle C, Jacobs JJ, Rosenberg AG. Outpatient total knee arthroplasty with a minimally invasive technique. J. Arthroplasty. 2005 ; 20(7) : 33-8.

13. Berger RA, Kusuma SK, Sanders SA, Thill ES, Sporer SM. The feasibility and perioperative complications of outpatient knee arthroplasty. Clin. Orthop. Relat. Res. 2009 ; 467(6) : 1443-9.

14. Bovonratwet P, Ondeck NT, Nelson SJ, Cui JJ, Webb ML, Grauer JN. Comparison of Outpatient vs Inpatient Total Knee Arthroplasty: An ACS-NSQIP Analysis. J. Arthroplasty. 2017 ; 32(6) : 1773-8. 
15. Lovald ST, Ong KL, Malkani AL, Lau EC, Schmier JK, Kurtz SM, et al. Complications, mortality, and costs for outpatient and short-stay total knee arthroplasty patients in comparison to standard-stay patients. J. Arthroplasty. $2014 ; 29(3): 510-5$.

16. Arshi A, Leong NL, D'Oro A, Wang C, Buser Z, Wang JC, et al. Outpatient total knee arthroplasty is associated with higher risk of perioperative complications. J. Bone Jt. Surg Am. 2017 ; 99(23) : 1978-86.

17. Crawford DA, Scully W, McFadden L, Manoso M. Preoperative Predictors of Length of Hospital Stay and Discharge Disposition Following Primary Total Knee Arthroplasty at a Military Medical Center. Mil. Med. 2011 ; 176(3) : 304-7.

18. Ansari D, Gianotti L, Schröder J, Andersson R. Fasttrack surgery : Procedure-specific aspects and future direction. Langenbeck's Arch. Surg. 2013 ; 398(1) : 29-37.

19. Krause A, Sayeed Z, El-Othmani M, Pallekonda V, Mihalko W, Saleh KJ. Outpatient Total Knee Arthroplasty : Are We There Yet? (Part 1). Orthop. Clin. North Am. 2018 ; 49(1) : 1-6.
20. Gromov K, Kjærsgaard-Andersen P, Revald P, Kehlet H, Husted H. Feasibility of outpatient total hip and knee arthroplasty in unselected patients : A prospective 2-center study. Acta Orthop. 2017 ; 88(5) : 516-21.

21. Hirschmann MT, Kort N, Kopf S, Becker R. Fast track and outpatient surgery in total knee arthroplasty : beneficial for patients, doctors and hospitals. Knee Surgery, Sport Traumatol. Arthrosc. 2017 ; 25(9) : 2657-8.

22. Kort NP, Bemelmans YFL, van der Kuy PHM, Jansen J, Schotanus MGM. Patient selection criteria for outpatient joint arthroplasty. Knee Surgery, Sport Traumatol. Arthrosc. 2017 ; 25(9) : 2668-75.

23. Husted H, Holm G, Jacobsen S. Predictors of length of stay and patient satisfaction after hip and knee replacement surgery: Fast-track experience in 712 patients. Acta Orthop. $2008 ; 79(2)$ : 168-73.

24. Krause A, Sayeed Z, El-Othmani M, Pallekonda V, Mihalko W, Saleh KJ. Outpatient Total Knee Arthroplasty : Are We There Yet? (Part 2). Orthop. Clin. North Am. 2018 ; 49(1) : 7-16. 